\title{
RISCO DE SOBREPESO E OBESIDADE EM SOLDADOS DO CORPO DE BOMBEIROS
}

\author{
Lúcio Kerber Canabarro \\ Universidade Federal de Pelotas, Pelotas, Rio Grande do Sul, Brasil \\ Airton José Rombaldi \\ Universidade Federal de Pelotas, Pelotas, Rio Grande do Sul, Brasil
}

\begin{abstract}
Resumo
O estudo objetivou avaliar o risco de obesidade e obesidade visceral de soldados do Corpo de Bombeiros de Pelotas - RS e relacionar com o tempo de serviço. O índice de massa corporal (IMC), e o perímetro abdominal (PA) foram coletados de todos os soldados ativos do batalhão. Os sujeitos foram divididos em G1 - soldados com menos de cinco anos de serviço $(n=14)$ e $\mathrm{G} 2$ - soldados com mais de cinco anos de serviço $(n=33)$. O grupo G1 apresentou tempo de serviço de $1,9( \pm 1,3)$ anos, IMC de $23,6( \pm 3,7) \mathrm{kg} / \mathrm{m} 2$ e PA de $84,2( \pm 8,5) \mathrm{cm}$. O grupo G2 teve tempo de serviço de $15,4( \pm 5,9)$ anos, IMC de $27,5( \pm 3,9) \mathrm{Kg} / \mathrm{m} 2$ e PA de $97,1 \mathrm{~cm}( \pm 9,8)$. Concluiu-se que os bombeiros mais antigos apresentaram maiores indicadores de obesidade, estando mais expostos aos riscos relacionados ao excesso de gordura corporal.
\end{abstract}

Palavras-chave: IMC - Gordura Abdominal - Obesidade - Bombeiros

\section{Introdução}

dieta inadequada e a inatividade física compõem um complexo
de causas de grande importância para a saúde da população. Estes fatores se associam fortemente a muitas doenças crônicas não transmissíveis e altamente prevalentes, a exemplo da obesidade, diabetes tipo 2 e doença coronariana (WHO, 2003).

O sobrepeso e a obesidade além de afetar a capacidade cardiorrespiratória e musculoesquelética e de favorecer o aumento da morbidade por doenças crônicas como as cardiopatias, o diabetes e a depressão, podem representar risco ou limitação para o desempenho das atividades ocupacionais, em especial em atividade com exigências físicas importantes (LAITINEN et al. 2005; MARTINEZ; LATORRE, 2008). Nesta esfera, destaca-se o trabalho de servidores públicos que 
prestam serviços essenciais como os policiais e os bombeiros (LALIĆ; BUKMIR; FERHATOVIĆ, 2007).

A forma de ingresso destes servidores na função de socorristas se dá através de concursos públicos, os quais têm por princípio selecionar os mais aptos e capacitados para desempenhar o cargo. Tratando de serviços de policiamento e salvamento, as provas, além de teóricas, abrangem etapas ditas físicas, nas quais o indivíduo é testado em suas capacidades físicas aeróbias e anaeróbias, além de destrezas específicas de cada cargo (SECRETARIA DE JUSTIÇA E SEGURANÇA DO RIO GRANDE DO SUL, 2009). Esta etapa física tem caráter eliminatório e geralmente o desempenho exigido é relativamente alto, considerando a população em geral (SECRETARIA DE JUSTIÇA E SEGURANÇA DO RIO GRANDE DO SUL, 2009).

Porém, no Brasil, uma vez que tais policiais ou bombeiros ingressaram no serviço não são mais avaliados fisicamente. Parte-se do pressuposto de que a condição física destes indivíduos permanecerá inalterada perpetuamente, premissa equivocada já que o exercício não tem o poder de deixar alterações fisiológicas permanentes. Quando cessam os estímulos, desaparecem as adaptações (SLENTZ et al., 2007). Especificamente em relação a essa população, há estudos mostrando que bombeiros apresentam a tendência de engordar e reduzir sua aptidão física à medida que envelhecem (CARVALHO et al., 2007; LALIĆ; BUKMIR; FERHATOVIĆ, 2007).

Nesse sentido, esse estudo objetivou verificar o risco de obesidade e de obesidade visceral de um dos setores do funcionalismo, o corpo de bombeiros da cidade de Pelotas - RS. Secundariamente, buscou-se investigar a relação entre o tempo de serviço e o risco de obesidade nos soldados, averiguando assim a efetiva repercussão do teste físico na carreira desses profissionais.

\section{Métodos}

Trata-se de um estudo transversal, de caráter descritivo. Todos os participantes foram informados previamente sobre os objetivos e procedimentos e desdobramentos futuros da pesquisa e assinaram um termo de consentimento livre e esclarecido. A pesquisa foi aprovada pelo Comitê de Ética da Escola Superior de Educação Física da Universidade Federal de Pelotas (Protocolo 024/2008).

Participaram do estudo todos os soldados do corpo de bombeiros 
da cidade de Pelotas - RS, em serviço no período de novembro e dezembro de 2008, totalizando um número de 47 sujeitos, os quais foram divididos em dois grupos: G1 composto com soldados com menos de cinco anos de serviço $(\mathrm{n}=14)$ e, $\mathrm{G} 2$ formado por soldados com cinco anos ou mais de serviço $(n=33)$. A divisão dos grupos desta forma ocorreu em função de uma série de fatores. O primeiro deles é que todos os soldados são submetidos no período de formação a treinamento físico na Academia Militar em Porto Alegre - RS; dessa forma, espera-se que nos primeiros anos de serviço as condições físicas sejam melhores tanto por este treinamento como pelas exigências do estágio probatório. Em segundo lugar, porque cinco anos é um tempo razoável para modificação na composição corporal de um indivíduo, sendo que após esse período se acentuam as licenças de saúde na corporação. Em terceiro lugar, está o fato de que muitos soldados acabam subindo na hierarquia militar com o passar dos anos, e assim, a escolha de tempo de serviço muito grande, oito anos ou mais, acabaria por limitar muito o número de soldados integrantes desse grupo.

As coletas de dados foram realizadas nos períodos de troca de turno, a saber, oito e vinte horas; isto é, os sujeitos foram avaliados no momento em que ingressavam no seu expediente de trabalho. O processo de coleta tinha duração de aproximadamente cinco minutos por sujeito avaliado e teve a seguinte ordem de procedimentos: pergunta sobre o tempo de serviço e da idade do sujeito; medida da massa corporal, da estatura e, por fim, do perímetro abdominal.

Foram coletadas as seguintes medidas: massa corporal $(\mathrm{Kg})$, estatura $(\mathrm{m})$, perímetro abdominal $(\mathrm{cm})$, idade (anos) e tempo de serviço na profissão (meses). A massa corporal foi determinada com uma balança digital da marca Tanita, com precisão para $0,1 \mathrm{Kg}$. Todos os soldados foram medidos com a farda padrão (calça, camiseta e gandóla), bem como com o calçado (coturno). A escolha por realizar a medida sem despir os coturnos foi devido ao fato dos soldados estarem em serviço. Após, foi descontado $2 \mathrm{Kg}$ do valor da massa corporal obtido de cada soldado, por ser o peso médio dos coturnos.

A medida da estatura foi tomada através de estadiômetro especialmente construído para esse fim, sendo a medida tomada com precisão de $0,1 \mathrm{~cm}$. A verificação também foi realizada calculando-se a altura dos coturnos, tendo se descontado $2 \mathrm{~cm}$, altura média do solado do coturno, da estatura coletada em cada um dos soldados. Após esse procedimento, procedeu-se o cálculo do índice de massa corporal (IMC) 
e, indivíduos com valores variando entre 25,0 e $29,9 \mathrm{Kg} / \mathrm{m} 2$ foram considerados com sobrepeso e aqueles com IMC $\geq 30,0 \mathrm{Kg} / \mathrm{m} 2$ foram classificados como obesos (WHO, 1995).

O IMC tem sido amplamente utilizado em pesquisas epidemiológicas (MARINHO et al., 2003; GIGANTE et al., 2006). É capaz de indicar o estado nutricional de populações com um custo muito baixo, e possui uma alta praticidade. Apesar de não demonstrar informações a respeito da composição corporal, de ser equivocado o seu uso individual e de apresentar alguns problemas em determinadas populações como asiáticas, é um critério utilizado pela Organização Mundial de Saúde como uma ferramenta importante na verificação do alastramento da epidemia de obesidade no mundo. A própria instituição classifica como sujeitos adultos obesos aqueles com IMC $\geq 30 \mathrm{Kg} / \mathrm{m} 2$. Diversos estudos têm se debruçado sobre este tipo de avaliação, e há evidências demonstrando que há correlação entre a gordura corporal, o IMC e outros valores antropométricos importantes (PEIXOTO et al., 2006; SAMPAIO; FIGUEIREDO, 2005; GIGANTE et al., 2006).

O perímetro abdominal foi determinado por meio de fita métrica inextensível (marca Mabbis, modelo Gulick) no nível natural da cintura, ponto médio entre a crista ilíaca anterior superior e a última costela, com precisão de $0,1 \mathrm{~cm}$. Ressalta-se que todos os soldados foram avaliados vestidos com uma camisa de algodão e a gandola, e para se tentar reduzir possíveis superestimações se subtraiu $2 \mathrm{~cm}$ da medida verificada em cada um dos soldados. Todas as verificações foram realizadas uma única vez e o acúmulo de gordura na cintura, ou obesidade abdominal, foi classificado em dois níveis. O nível 1 correspondeu aos valores entre 94,0 e 101,9 cm; e o nível 2 correspondeu a valores $>102,0 \mathrm{~cm}$ sendo que o primeiro indicou risco cardiovascular moderado e o segundo, alto risco cardiovascular (WHO, 1995; CARNEIRO et al., 2003; PEIXOTO et al., 2006; SOCIEDADE BRASILEIRA DE CARDIOLOGIA, 2007). Valores abaixo de $94 \mathrm{~cm}$ de perímetro foram considerados adequados. $\mathrm{O}$ fato da massa corporal e da estatura terem sido medidas com os soldados vestidos, representa uma limitação do presente estudo.

O perímetro abdominal é uma medida antropométrica utilizada a fim de averiguar se o indivíduo está ou não em um grupo de risco. Sua lógica parte do pressuposto de que o perímetro desta região pode dar uma idéia da quantidade de gordura depositada, ou seja, uma gordura que provavelmente é visceral, sendo esta a mais relacionada com a 
síndrome metabólica e com a resistência a insulina (CARNEIRO et al., 2003).

$\mathrm{Na}$ análise estatística, para as variáveis contínuas foi empregado o teste de normalidade de Shapiro-wilk, que demonstrou que os dados apresentaram distribuição normal, exceto para a variável idade. Desta forma, foi empregado o teste " $t$ " para amostras independentes para as variáveis IMC, massa corporal e perímetro abdominal e o teste não pareado de Wilcoxon para a variável idade; os dados estão expressos como média \pm desvio-padrão. Quando as variáveis foram categóricas, se utilizaram o cálculo de proporções e o teste Exato de Fischer. O nível de significância aceito foi de $\mathrm{p} \leq 0,05$.

\section{Resultados}

Os resultados encontrados mostraram massa corporal média de $83,1 \mathrm{Kg}( \pm 14,6)$ e a estatura de $1,77 \mathrm{~m}( \pm 0,07)$. Dessa forma, o escore médio de IMC foi de $26,0 \mathrm{Kg} / \mathrm{m} 2( \pm 4,2)$, o perímetro abdominal de $93,3 \mathrm{~cm}( \pm 11,1)$, e a idade média de 33,9 anos $( \pm 8,0)$. Estes dados formaram um retrato do batalhão de soldados como um todo, isto é, sem subdivisões por tempo de serviço com a corporação.

No entanto, como se pode observar na tabela 1, quando separados em grupos considerando o tempo de serviço, houve diferenças estatisticamente significativas para as variáveis: tempo de serviço, idade, massa corporal, IMC e perímetro abdominal; sendo o G2 (mais antigo na carreira), o grupo com piores indicadores antropométricos.

Tabela 1: Valores médios ( \pm DP) das variáveis: tempo de serviço, idade, massa corporal, estatura, IMC e perímetro abdominal de ambos os grupos $(n=47)$

\begin{tabular}{lcc}
\hline \multicolumn{1}{c}{ V ariáveis } & $\mathrm{G}_{1}$ & $\mathrm{G}_{2}$ \\
\hline Tempo de serviço (meses) & $23,3( \pm 16,2)$ & $185,6( \pm 71,3)^{*}$ \\
Idade (anos) & $24,3( \pm 3,3)$ & $38,0( \pm 5,4)^{*}$ \\
Massa corporal $(\mathrm{Kg})$ & $75,4( \pm 15,9)$ & $86,4( \pm 12,9)^{* *}$ \\
Estatura $(\mathrm{cm})$ & $178( \pm 8,3)$ & $177( \pm 6,5)$ \\
IMC $\left(\mathrm{Kg} / \mathrm{m}^{2}\right)$ & $23,6( \pm 3,7)$ & $27,5( \pm 3,9)^{* * *}$ \\
Perim etro abdominal $(\mathrm{cm})$ & $84,2( \pm 8,5)$ & $97,9( \pm 9,9)^{*}$ \\
\hline
\end{tabular}

${ }^{*} \mathrm{p}<0,001 ; * * \mathrm{p} \leq 0,01 ; * * * \mathrm{p}<0,05$

A tabela 2 apresenta as associações entre tempo de serviço dos soldados e o risco de sobrepeso e obesidade, por meio do IMC, e o risco de obesidade visceral, por meio do perímetro abdominal. Pode-se observar, para ambas as variáveis de risco de obesidade, que à medida que os sujeitos ultrapassam o tempo de cinco anos na carreira aumen- 
ta significativamente o risco de sobrepeso/obesidade e obesidade visceral. Em relação ao IMC, verifica-se que dos 24 sujeitos que apresentam sobrepeso/obesidade, $21(85,5 \%)$ estão no grupo que está a mais de cinco anos no serviço militar. Resultado similar se verifica no tocante ao risco de obesidade visceral, onde dos 19 soldados nas condições de risco níveis 1 e 2, $17(89,5 \%)$ pertencem ao grupo que está a mais tempo em serviço.

Tabela 2: Associação entre o tempo de serviço e as variáveis IMC e perímetro abdominal $(\mathrm{n}=47)$

\begin{tabular}{|c|c|c|c|c|c|}
\hline \multirow[t]{3}{*}{ V ariáveis } & \multicolumn{4}{|c|}{ Tempo de serviço } & \multirow[t]{3}{*}{ V alor $p$} \\
\hline & \multicolumn{2}{|c|}{$<5$ anos } & \multicolumn{2}{|c|}{$\geq 5$ anos } & \\
\hline & $\mathrm{n}$ & $\%$ & $\mathrm{n}$ & $\%$ & \\
\hline IMC $\left(\mathrm{Kg} / \mathrm{m}^{2}\right)$ & & & & & $0,03 *$ \\
\hline Eutrófico & 11 & 47,8 & 12 & 52,2 & \\
\hline Sobrepeso & 2 & 14,3 & 12 & 85,7 & \\
\hline Obesidade & 1 & 10,0 & 9 & 90,0 & \\
\hline \multicolumn{6}{|c|}{ Perím etro abdominal $(\mathrm{cm})$} \\
\hline Adequado & 12 & 42,9 & 16 & 57,1 & $0,05^{*}$ \\
\hline Risco nivel 1 & 1 & 14,3 & 6 & 85,7 & \\
\hline Risco nivel 2 & 1 & 8,3 & 11 & 91,7 & \\
\hline
\end{tabular}

* Teste exato de Fisher

\section{Discussão}

Os resultados indicaram que uma parte considerável do batalhão $(29,8 \%)$ encontrou-se na zona de sobrepeso, e pouco mais de $1 / 5$ $(21,3 \%)$, alcançou o ponto de corte para obesidade, segundo o critério utilizado para o IMC. A repercussão disto é preocupante, pois sujeitos com valores de IMC menores que $30 \mathrm{Kg} / \mathrm{m} 2$, já podem estar apresentando sintomas de hipertensão arterial, uma doença potencialmente letal. Os resultados do presente estudo foram similares a outros publicados em diferentes países (MORIOKA; BROWN, 1970; KALES et al., 1999; LALIĆ; BUKMIR; FERHATOVIĆ, 2007; CARVALHO et al., 2007), indicando que à medida que os bombeiros se tornam mais velhos, maior é o risco de se encontrarem com sobrepeso e/ou obesidade. Um fator que poderia explicar valores altos do IMC seria uma proporção de massa muscular avantajada, a qual tem uma densidade maior que a massa gorda, o que poderia levar tais indivíduos a serem classificados como obesos quando na verdade apresentariam taxa proporcionalmente maior de músculos do que de gordura. Este parece não ter sido o caso no presente estudo, visto que se fosse 
assim, os valores do perímetro abdominal seriam menores, e o que ocorreu, corroborou para dar crédito ao IMC encontrado.

Quanto ao perímetro abdominal, os valores encontrados neste estudo revelam que $25,5 \%$ dos soldados avaliados se encontram na zona de alto risco para doença cardiovascular (risco nível 2), servidores que estão sob um risco eminente de passarem do papel de socorristas para socorridos. Já os soldados que estiveram entre 94 e $102 \mathrm{~cm}$ totalizaram $14,9 \%$. Somados os dois grupos, aproximadamente $40 \%$ dos sujeitos estão em uma condição de risco cardiovascular importante, o que é alarmante. Os resultados do presente estudo, no entanto, são similares aos relatados por Carvalho et al. (2007), os quais conduziram um estudo com soldados do corpo de bombeiros no estado do Ceará.

É interessante ressaltar que há a possibilidade de que os pontos de corte adotados no perímetro abdominal possam subestimar a exposição a risco cardíaco em determinadas etnias. Dessa forma, negros parecem ser mais suscetíveis a complicações cardíacas e apresentam riscos metabólicos maiores que os brancos mesmo que tenham a mesma quantidade de gordura visceral (CARNEIRO et al., 2003). Assim, Barbosa et al. (2006) em consequência de seu estudo na população da cidade de Salvador - BA, sugerem ponto de corte $>88 \mathrm{~cm}$, ao invés de $\geq 102 \mathrm{~cm}$, para a população brasileira.

Outros estudos avaliaram a correlação do perímetro abdominal e do IMC com a hipertensão arterial (CARNEIRO et al., 2003; SAMPAIO; FIGUEIREDO, 2005; SARNO; MONTEIRO, 2007). Houve, no entanto, quem tenha indicado que o ponto de corte adotado de 102 $\mathrm{cm}$ para perímetro abdominal e de $30 \mathrm{Kg} / \mathrm{m}^{2}$ no IMC, não são adequados para identificar grupos com maior risco de pressão alta, sendo que os valores que melhor identificaram este grupo, para homens, foram inferiores a $94 \mathrm{~cm}$ e de $25 \mathrm{Kg} / \mathrm{m}^{2}$ (PEIXOTO et al. 2006). Isso pode significar que uma grande parcela dos soldados avaliados, cerca de $50 \%$, pode encontrar-se em um grupo de risco para hipertensão, um dos componentes que posteriormente pode conduzir a doença cardiovascular (ADAMS et al., 2006; LITWIN, 2008).

No presente estudo observou-se que os sujeitos do G1, mais jovens, apresentaram valores estatisticamente menores para IMC, peso corporal e perímetro abdominal que os soldados do G2. Os soldados do corpo de bombeiros, que serviram de amostra para esse estudo, dessa forma, não se diferenciaram da população da cidade, pois estudos epidemiológicos de base populacional conduzidos no município 
de Pelotas - RS apontaram que há um aumento considerável no nível de sedentarismo conforme se aumenta a idade, sendo que o grupo entre 20-29 anos é mais ativo com relação às faixas etárias mais avançadas (HALLAL et al., 2005a; HALLAL et al., 2005b; GIGANTE et al., 2006). Além da diferença etária já mencionada, os mais jovens estiveram a menos tempo no curso de formação de Bombeiros da Academia da Brigada Militar, na cidade de Porto Alegre - RS, onde as exigências físicas são severas, e isso provavelmente, influenciou sua condição física e seus escores antropométricos. Outro dado que provavelmente contribuiu para os menores escores dos sujeitos do G1 é o estágio probatório, com duração de três anos e obrigatório para o ingresso no serviço público. Nesse sentido, o fato de estar sendo avaliado talvez exerça influência positiva na manutenção da condição física.

As prevalências apresentadas nesse estudo com militares, em relação ao risco de obesidade e obesidade visceral, foram similares aos encontrados na população de Pelotas - RS como um todo, onde 50\% da população estão com sobrepeso ou algum grau de obesidade (GIGANTE et al., 2006; NEUTZLING et al. 2009; CORRÊA et al., 2010), escores que demonstram a necessidade de medidas urgentes pois são incompatíveis com a função de bombeiro.

O último edital para concurso público para o provimento de vagas para soldado do corpo de bombeiros no Estado do Rio Grande do Sul (SJS - RS, 2005) estabeleceu que, na prova física, para serem aprovados, entre outras exigências, os candidatos do sexo masculino deverão ser capazes de percorrer, no tempo de 12 minutos uma distância mínima de 2600 metros, o que é equivalente a um escore de consumo máximo de oxigênio de $44 \mathrm{~mL} / \mathrm{Kg} . \mathrm{min}$; isto é, uma faixa classificada em algumas tabelas de como sendo "bom" ou "recomendado" para homens com idade entre 20-35 anos (COOPER, 1972). É importante salientar que o índice exigido nos editais geralmente não é alcançado por candidatos que estejam com excesso de gordura corporal. Os resultados encontrados para o grupo G2 apontam para o fato de que a condição físico-antropométrica destes soldados deteriorou-se no decorrer da carreira e, cerca de $50 \%$ do batalhão, por critérios estabelecidos pela própria Brigada Militar, não está apta para desempenhar a função que exercem, e destes $50 \%$, sequer $30 \%$ realizariam a prova física exigida para ingresso na carreira, pois seriam previamente eliminados por não atenderem os valores de IMC fixados nos editais.

Assim, são necessárias mudanças nos critérios de avaliação dos 
servidores públicos que dependem da condição física para melhor desempenhar suas funções a fim de beneficiar tanto os próprios soldados - para que mantenham a condição físico-antropométrica que lhes é exigida quando da admissão no serviço público, o que por sua vez será um fator importante de proteção as doenças crônico-degenerativas quanto o Estado sob o aspecto financeiro, já que haveria uma redução de custos com assistência médico-hospitalar e também nos custos decorrentes com os afastamentos de soldados.

\title{
Conclusão
}

Concluiu-se que os soldados do corpo de bombeiros da cidade de Pelotas - RS apresentam elevado risco para sobrepeso, obesidade e obesidade visceral, considerados de risco para o desenvolvimento de doenças crônico-degenerativas. Tais fatos revelam a exposição dos socorristas aos riscos relacionados à saúde o que pode dificultar o cumprimento das mais variadas missões em presença de situações que envolvam também o socorrido.

Políticas do governo do Estado e estratégias do comando do batalhão do corpo de bombeiros, envolvendo cuidados alimentares e prática regular de atividade física para sanar este problema tão pertinente que afeta diretamente toda a população, são necessárias.

Estudos futuros deviam considerar intervenções com prática de atividade física e cuidados alimentares, especialmente no ambiente do trabalho, no sentido de modificar o quadro de sobrepeso e obesidade nessa população.

\section{Risk of overweight and obesity for firefighters}

\begin{abstract}
In this study, we aimed at assessing the risk of obesity and visceral obesity in firefighters from Pelotas city and relate it to their service years. The body mass index (BMI) and waist circumference (WC) were collected from all active firefighters. The subjects were divided into G1 - firefighters with less than five years of service $(n=14)$ and G2 - firefighters with more than five years of service $(n=33)$. The group G1 presented service years of $1.9( \pm 1.3)$, BMI of $23.6( \pm 3.7) \mathrm{kg} / \mathrm{m} 2$, and WC of $84.2( \pm 8.5) \mathrm{cm}$. The group G2 had service years of $15.4( \pm 5.9)$, BMI 27.5 $( \pm 3.9) \mathrm{kg} / \mathrm{m} 2$, and $\mathrm{WC}$ of $97.1( \pm 9.8) \mathrm{cm}$. We can say that the older firefighters had higher levels of obesity, being more exposed to risks related to excess body fat.

Keywords:BMI - Abdominal Fat - Obesity - Firefighters
\end{abstract}


Riesgo de Sobrepeso y Obesidad de Soldados de la Corporación de Bomberos

\section{Resumen}

El objetivo del estudio fue evaluar el riesgo de obesidad y obesidade visceral de la Corporación de Bomberos de Pelotas-RS y correlacionar con la duración del tiempo de servicio de la profesión. El índice de masa corporal (IMC) y la circunferencia abdominal fueron colectadas de todos los soldados activos de la corporación. Los sujetos fueron divididos en G1 - soldados con menos de cinco años de servicio $(n=14)$ y G2 - soldados con más de cinco años de servicio $(n=33)$. El grupo G1 tuvo tiempo de ejercicio de 1,9 $( \pm 1,3)$ años, IMC de $23,6( \pm 3,7) \mathrm{Kg} / \mathrm{m} 2$ y la CC de $84,2( \pm 8,5) \mathrm{cm}$. E1 grupo G2 ha tenido tiempo de ejercicio de $15,4( \pm 5,9)$ años, IMC de 27,5 $( \pm 3,9) \mathrm{Kg} / \mathrm{m} 2$ y la CC de 97,1 $( \pm 9,8) \mathrm{cm}$. Se concluyó que los bomberos más viejos, tuvieron mayores indicadores de obesidad, estándo más expuestos a riesgos relacionados con lo exceso de grasa corporal.

Palabras clave: IMC - Grasa Abdominal - Obesidad - Bomberos

\section{Referências}

ADAMS, K. F. et al. Overweight, obesity, and mortality in a large prospective cohort of persons 50 to 71 years old. New England Journal of Medicine, Boston-MA, v. 355, p. 763-778, 2006.

BARBOSA, P. J. B. et al. Critério de obesidade central em população brasileira: impacto sobre a síndrome metabólica. Arquivos Brasileiros de Cardiologia, Rio de Janeiro, v. 87, p. 407-412, 2006.

CORREAA, L. Q. et al. Aging male's symptoms in a southern Brazil population: lifestyle effects after the age of 40. The Aging Male, New York, v. 13, p. 93-99, 2010.

COOPER, K. Capacidade aeróbica. 2. ed. Rio de Janeiro: Fórum, 1972.

CARNEIRO, G. et al. Influência da distribuição da gordura corporal sobre a prevalência de hipertensão arterial e outros fatores de risco cardiovascular em indivíduos obesos. Revista da Associação Médica Brasileira, São Paulo, v. 49, n. 3, p. 306-311, 2003.

CARVALHO, L. N. et al. Níveis de composição corporal e risco cardíaco por perimetria de bombeiros militares na região do cariri cearense, Brasil. Anais do XXIV Congresso Nacional de Atividade Física e Fisioterapia - CONAFF, Fortaleza-CE, p. 112-113, 2007. 
GIGANTE, D. P. et al. Obesidade da população adulta de Pelotas, Rio Grande do Sul, Brasil e associação com nível sócio-econômico. Cadernos de Saúde Pública, Rio de Janeiro, v. 22, n. 9, p. 1873-1879, 2006.

HALLAL, P. C. et al. Who, when, and how much? Epidemiology of walking in a middle-income country. American Journal of Preventive Medicine, New York, v. 28, n. 2, p. 156-161, 2005 a.

HALLAL P. C. et al. Physical activity in adults from two Brazilian areas: similarities and differences. Cadernos de Saúde Pública, Rio de Janeiro, v. 21, n. 2, p. 573-580, 2005 b.

KALES, S. N. et al. Correlates of body mass index in hazardous materials firefighters. Journal of Occupational and Environmental Medicine, Munbai, v. 41, n. 7, p. 589-595, 1999.

LAITINEN, J. et al. Body size and perceived work ability - results from the Northern Finland 1966 and 1986 birth cohorts. International Symposium on Youth and Work Culture, Tampere-Finland, 2005, Espoo.

LALIĆ, H.; BUKMIR, L.; FERHATOVIĆ, M. Simulation of working conditions by maximum work load on firefighters. Collegium Antropologicum, Zagreb-Croatia, v. 31, n. 1, p. 153-158, 2007.

LITWIN, S. E. Which measures of obesity best predict cardiovascular risk? Journal of the American College of Cardiology, San DiegoCA, v. 52, n. 8, p. 616-619, 2008.

MARINHO, S. P. et al. Obesidade em adultos de segmentos pauperizados da sociedade. Revista de Nutrição, Campinas-SP, v. 16 n. 2, p. 195-201, 2003.

MARTINEZ, M. C.; LATORRE, M. R. D. O. Saúde e capacidade para o trabalho de eletricitários do Estado de São Paulo. Ciência e Saúde Coletiva, Rio de Janeiro, v. 13, n. 6, p. 1061-1073, 2008.

MORIOKA, H. M.; BROWN, M. L. Incidence of obesity and overweight among honolulu police and firemen. Public Health Reports, Boston, v. 85, n. 5, p. 433-440, 1970.

NEUTZLING, M. B. et al. Fatores associados ao consumo de frutas, 
legumes e verduras em adultos de uma cidade no Sul do Brasil. Cadernos de Saúde Pública, Rio de Janeiro, v. 25, n. 11, p. 2365-2374, 2009.

PEIXOTO, M. R. G. et al. Circunferência da cintura e índice de massa corporal como preditores da hipertensão arterial. Arquivos Brasileiros de Cardiologia, Rio de Janeiro, v. 87, p. 462-470, 2006.

SAMPAIO, L. R.; FIGUEIREDO, V. O. C. Correlação entre o índice de massa corporal e os indicadores antropométricos de distribuição de gordura corporal em adultos e idosos. Revista de Nutrição, CampinasSP, v. 18, n. 1, p. 53-61, 2005.

SARNO, F.; MONTEIRO, A. A. Importância relativa do índice de Massa Corporal e da circunferência abdominal na predição da hipertensão arterial. Revista de Saúde Pública, São Paulo, v. 41, n. 5, p. 788796, 2007.

SJS-RS - SECRETARIA DE JUSTIÇA E SEGURANÇA DO RIO GRANDE DO SUL. Edital 01/2005 - Concurso público para o cargo de militar estadual na graduação de soldado. 2005. Disponível em: http://www.fdrh.rs.gov.br/editais/Brigada2005/EditalBrigadaMilitar_N01.pdf. Acesso em: 30 outubro 2009.

SLENTZ, C. A. et al. Inactivity, exercise training and detraining, and plasma lipoproteins. STRRIDE: a randomized, controlled study of exercise intensity and amount. Journal of Applied Physiology, Bethesta, v. 103, p. 432-442, 2007.

SOCIEDADE BRASILEIRA DE CARDIOLOGIA. IV Diretriz Brasileira Sobre Dislipidemias e Prevenção da Aterosclerose do Departamento de Aterosclerose. Arquivos Brasileiros de Cardiologia, Rio de Janeiro, v. 88, Suplemento 1, 2007.

WHO. Physical Status: the use and interpretation of anthropometry Report of a WHO Expert Committee. Geneva, WHO Technical Report Series 854, 1995.

WHO. FAO. Expert consultation on diet, nutrition and the prevention diseases. Diet nutrition and the prevention of chronic diseases: report of a joint WHO/FAO WHO expert consultation. Geneva, WHO Technical Report Series 916, 2003. 
Recebido em: 12/06/2010

Revisado em: 30/08/2010

Aprovado em: 18/09/2010

\section{Endereço para correspondência}

luciokerber@yahoo.com.br

Lúcio Kerber Canabarro

Universidade Federal de Pelotas

Escola Superior de Educação Física (ESEF)

Rua Luiz de Camões, 625 - Bairro Tablada

CEP: 96055-630 - Pelotas/RS - Brasil 